\title{
GSM Based Automatic Electricity Billing System
}

\author{
Kanthimathi ${ }^{1}$, Krishnammal $^{2}$, Jeyabala ${ }^{3}$, Jothi Monika ${ }^{4}$, Muthu Veni ${ }^{5}$, Christo Ananth ${ }^{6}$ \\ U.G.Scholars, Department of ECE, Francis Xavier Engineering College, Tirunelveli ${ }^{1,2,3,4,5}$ \\ Associate Professor, Department of ECE, Francis Xavier Engineering College, Tirunelveli ${ }^{6}$
}

\begin{abstract}
In this project an automatic meter reading system is designed using GSM Technology. The embedded micro controller is interfaced with the GSM Module. This setup is fitted in home. The energy meter is attached to the micro controller. This controller reads the data from the meter output and transfers that data to GSM Module through the serial port. The embedded micro controller has the knowledge of sending message to the system through the GSM module. Another system is placed in EB office, which is the authority office. When they send "unit request" to the microcontroller which is placed in home. Then the unit value is sent to the EB office PC through GSM module. According to the readings, the authority officer will send the information about the bill to the customer. If the customer doesn't pay bill on-time, the power supply to the corresponding home power unit is cut, by sending the command through to the microcontroller. Once the payment of bill is done the power supply is given to the customer. Power management concept is introduced, in which during the restriction mode only limited amount of power supply can be used by the customer.
\end{abstract}

Keywords: GSM, Automatic Meter reading system, Embedded Microcontroller

\section{INTRODUCTION}

The system consists of a microcontroller, GSM module, buzzer, LCD display and a relay switch. The system's microcontroller continuously reads the energy meter and the GSM transmitter transmits the information wirelessly to the GSM receiver which is connected to the computer at the EB Office. If the user pays the bill on time the power supply will be provided to the user without any interrupt. If the user fails to pay the bill the power supply to the user will be cut. Power management system is introduced in which during normal mode user can consume the amount of power he/she requires, but during the restriction mode only limited amount of power supply is given to the customer.

The service provider [1] for energy still uses conventional methods for getting the energy consumed by individual customer. This system automatically reads the energy consumed and sends it to the service provider using the existing short message service
(SMS).

The billing process of electricity consumption which we are using at present is very long process and requires lot of man power. For overcoming all the difficulties present in this system we are introducing fully automated billing process. Two GSM modems are used one connected to electric meter and another connected to PC present in office of electricity board. The PC is the computer which consists of all database such as meter reading, bill amount, due amount, last date for bill payment. GSM modem consists of SIM cards possessing unique number. User interface consists of LCD which displays the power consumed and amount of bill to be paid. User also consists of a mobile phone through which bill details can be communicated to the consumer. Bill can be paid just by recharging through the mobile.

The proposed system overcomes the disadvantages of the existing system such as errors which occurs due to manual meter reading, high man power consumption etc. The proposed system has the advantage of power disconnection and power reconnection. The 
power management system is introduced in which during the restriction mode only limited amount of power supply is provided to the customer, which reduces the scarcity of power during the period of power demand.

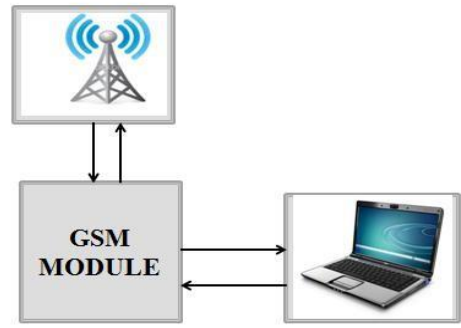

Fig.1. Block Diagram Of System Placed At EB

In the power management concept, two modes are being used, one is the normal mode and the other is the restricted mode. During normal mode, the customer can consume the power as much as it is required. During the power demand, the authority officer will select the restricted mode which limits the power supply to the each customer's house and sends the message to each customer's microcontroller via GSM. By receiving this message, the microcontroller alerts the customer with an alarm and controls the usage of power. If the customer tends to consume more power, then the controller automatically disconnects the whole power supply to that particular customer's house. The reconnection is made only after paying the fine to the government.

\section{SYSTEM DESIGN}

An easy way to comply with the conference paper formatting requirements is to use this document as a template and simply type your text into it.

The architecture of the 8051 family of microcontrollers (8051 derivatives) is referred to as the MCS-51 architecture (Micro Controller Series - 51), or sometimes simply as MCS-51. The block diagram of 89C51 microcontroller is shown below.

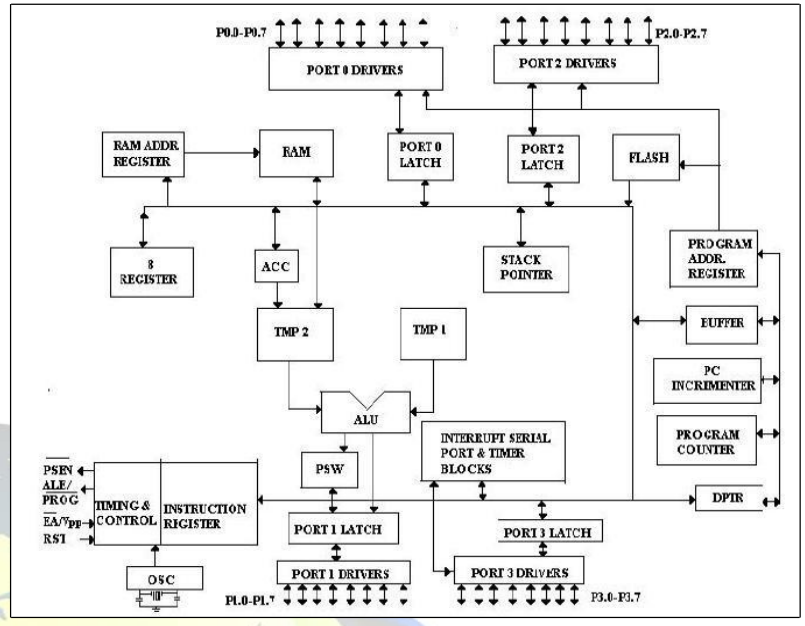

Fig.2. Architecture of 89C51 Microcontroller

Accumulator is a general-purpose register, which stores runtime results. Before performing any operation upon an operand, operand has to be stored in the accumulator. Results of arithmetical operations are also stored in the accumulator. When transferring data from one register to another, it has to go through the accumulator. Due to its versatile role, this is the most frequently used register, essential part of every MCU.

B Register is used along with the Accumulator for multiplication and division. This $\mathrm{B}$ register provides temporary storage space for the result of multiplication \& division operation. Instructions of multiplication and division can be applied only to operands located in registers $\mathrm{A}$ and $\mathrm{B}$. Other instructions can use this register as a secondary accumulator (A).

Term "port" refers to a group of pins on a microcontroller which can be accessed simultaneously, or on which we can set the desired combination of zeros and ones, or read from them an existing status. Ports represent physical connection of Central Processing Unit with an outside world. Microcontroller uses them in order to monitor or control other components or devices. 89C51 has 4 ports; with each port have 8-bit length. All the ports are bit and byte addressable.

Port 0 has two-fold role: If external memory is used, P0 behaves as address output (A0 - A7) when ALE pin is at high logical level, or as data output (Data Bus) when ALE pin is at low logical level, otherwise all bits of the port are either input or output. 
Another feature of this port comes to play when it has been designated as output. Unlike other ports, Port 0 lacks the "pull up" resistor (resistor with $+5 \mathrm{~V}$ on one end). This seemingly insignificant change has the following consequences:

- When designated as input, pin of Port 0 acts as high impedance offering the infinite input resistance with no "inner" voltage.

- When designated as output, pin acts as "open drain". Clearing a port bit grounds the appropriate pin on the case $(0 \mathrm{~V})$. Setting a port bit makes the pin act as high impedance. Therefore, to get positive logic $(5 \mathrm{~V})$ at output, external "pull up" resistor needs to be added for connecting the pin to the positive pole. Therefore, to get one $(5 \mathrm{~V})$ on the output, external "pull up" resistor needs to be added for connecting the pin to the positive pole.

Port 1 is $\mathrm{I} / \mathrm{O}$ port. Having the "pull up" resistor, Port 1 is fully compatible with TTL circuits.

When using external memory, this port $\mathrm{P} 2$ contains the higher address byte (addresses A8-A15), similar to Port 0 . Otherwise, it can be used as universal I/O port.

Beside its role as universal I/O port, each pin of Port 3 has an alternate function. In order to use one of these functions, the pin in question has to be designated as input, i.e. the appropriate bit of register P3 needs to be set. By selecting one of the functions the other one is disabled. From a hardware standpoint, Port 3 is similar to Port 0 .

The Data pointer register is made up of two 8 bit registers, named DPH (Data Pointer High) and DPL (Data Pointer Low). These registers are used to give addresses of the internal or external memory. The DPTR is under the control of program. DPTR is also manipulated as one 16 bit register, DPH \& DPL are each assigned an address. The 89C51 microcontroller has additional DPTR. The dual DPTR structure is a way by which the chip will specify the address of an external data memory location. There are two 16-bit DPTR registers that address the external memory, and a single bit called DPS (bit0 in AUXR1) that allows the program code to switch between them.
The stack refers to an area of internal RAM that is used in conjunction with certain opcodes to store and retrieve data quickly. The register used to access the stack is called Stack Pointer. The 8 bit stack pointer register is used by the 89C51 to hold an internal RAM address that is called then top of the stack. The stack pointer increments before storing the data on the stack. As retrieved from the stack the SP is decremented by one. The number in Stack Pointer points to the location of the last "valid" address within the Stack. With the beginning of every new routine, Stack Pointer increases by 1; upon return from routine, SP decreases by 1 . Upon reset (or turning the power on), the stack pointer contains the value $07 \mathrm{~h}$.

Program Counter is used to access code memory. Program counter always points to the address of the next instruction in memory to be executed. Upon reset (or turning the power on), the program counter resets to the starting location of the program.

Instruction Register: When an instruction is fetched from the Flash memory, it is loaded in the instruction register.

The timing and control unit synchronizes the operation of the microcontroller and generates control signals necessary for communication between the microcontroller and the peripherals.

The Program Status Word (PSW) register is an 8 bit register. It is also referred to as the flag register. It contains the math flags, user program flag F0, and the register select bits that identify which of the four general purpose register banks is currently in use by the program.

Oscillator circuit is used for providing a microcontroller with a clock. Clock is needed so that microcontroller could execute a program or program instructions. Stable pace provided by the oscillator allows harmonious and synchronous functioning of all other parts of MCU. The manufacturers make available 89C51 designs that can run at specified maximum and minimum frequencies, typically 1 megahertz to 33 megahertz. Minimum frequencies imply that some internal memories are dynamic and must always operate above a minimum frequency or data will be lost.

An interrupt is a signal from a device 
attached to a computer or from a program within the computer that causes the main program that operates the computer to stop and points out what to do next. In general, there are hardware interrupts and software interrupts. A hardware interrupt is related to the hardware of the system. For example, when an I/O operation is completed such as reading some data into the computer from a keyboard interrupt the main program. As the name implies the software interrupts related to the software of the system. It occurs when an application program terminates or requests certain services from the operating system.

Timers are usually the most complicated parts of a microcontroller. Physically, timer is a register whose value is continually increasing to FFFFh, and then it starts all over again: $0 \mathrm{~h}, 1 \mathrm{~h}, 2 \mathrm{~h}, 3 \mathrm{~h}, 4 \mathrm{~h} . . . \mathrm{FFFFh} . . .0 \mathrm{~h}, 1 \mathrm{~h}$, $2 \mathrm{~h}, 3 \mathrm{~h}$......etc. The 89C51 MCU clock employs a quartz crystal. As this frequency is highly stable and accurate, it is ideal for time measuring. Since one instruction takes 12 oscillator cycles to complete, the math is easy. 89C51 has three Timers/Counters marked as T0, T1 \& T2. Their purpose is to measure time and count external occurrences, but can also be used as clock in serial communication purpose called as, Baud Rate.

RAM is used for storing temporary data and auxiliary results generated during the runtime. The P89C51RD2BN has internal data memory that is mapped into four separate segments: the lower 128 bytes of RAM, upper 128 bytes of RAM, 128 bytes Special Function Register (SFR), and 768 bytes expanded RAM (ERAM).

The four segments are:

- The Lower 128 bytes of RAM (addresses $00 \mathrm{H}$ to $7 \mathrm{FH}$ ) are directly and indirectly addressable.

- The Upper 128 bytes of RAM (addresses $80 \mathrm{H}$ to FFH) are indirectly addressable only.

- The Special Function Registers, SFRs, (addresses $80 \mathrm{H}$ to FFH) are directly addressable only.

- The 768-bytes expanded RAM (ERAM, 00H 2FFH) are indirectly accessed by move external instruction, MOVX, and with the EXTRAM bit in the AUXR register cleared.
89C51 have built-in 64-kilo bytes of Flash memory. The P89C51RD2BN Flash memory augments EPROM functionality with in-circuit electrical erasure and programming. The Flash can be read and written as bytes. The Chip Erase operation will erase the entire program memory. The Block Erase function can erase any Flash block. In-system programming and standard parallel programming are both available. On-chip erase and write timing generation contribute to a user-friendly programming interface. The P89C51RD2BN Flash reliably stores memory contents even after 10,000 erase and program cycles. The cell is designed to optimize the erase and programming mechanisms. In addition, the combination of advanced tunnel oxide processing and low internal electric fields for erase and programming operations produces reliable cycling. The P89C51RD2BN uses a $+5 \mathrm{~V}$ VPP supply to perform the Program/Erase algorithms. LCD is used in widespread applications replacing LEDs (Seven segment LEDs or other multisegment LEDs) nowadays.

The LCD to latch information presented to its data pins uses the enable pin. When data is supplied to data pins, a high to low pulse must be applied to this pin in order for the LCD to latch in the data present at the data pins.

D0 - D7: The 8-bit data pins, are used to send information to the LCD or read the contents of the LCD's internal registers. To display letters and numbers, we send ASCII codes for the letters A-Z, a-Z, and numbers $0-9$ to these pins while making $\mathrm{RS}=1$.

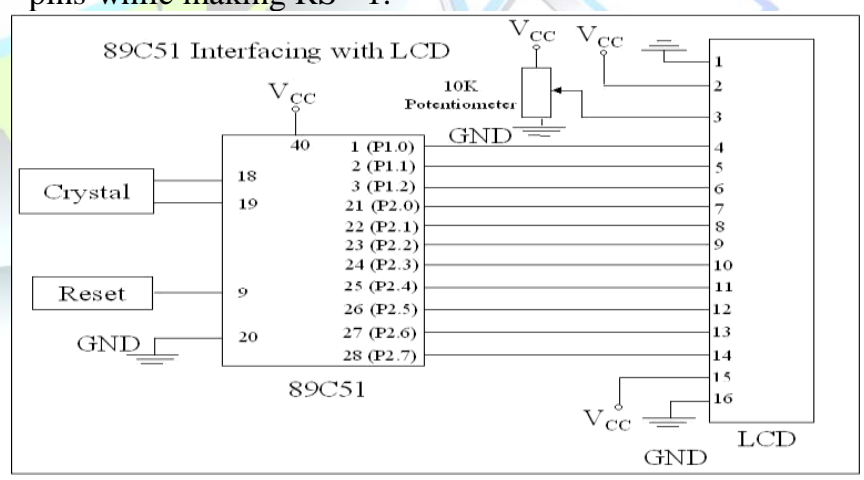

Fig.3. 89c51 Interfacing With LCD

A relay is an electrically operated switch. 
Many relays use an electromagnet to operate a switching mechanism mechanically, but other operating principles are also used. Relays are used where it is necessary to control a circuit by a low-power signal (with complete electrical isolation between control and controlled circuits), or where several circuits must be controlled by one signal. The first relays were used in long distance telegraph circuits, repeating the signal coming in from one circuit and re-transmitting it to another. Relays were used extensively in telephone exchanges and early computers to perform logical operations.

\section{III.PCB LAYOUT DESIGN}

GSM consists of Mobile Equipment (ME) such as hand portable and vehicle mounted unit Subscriber Identity Module (SIM), which contains the entire customer related information (identification, secret key for authentication, etc.)

It consist of Base Transceiver Station (BTS) defines a cell and is responsible for radio link protocols with the Mobile Station

Base Station Controller (BSC) controls multiple BTSs and manages radio channel setup, and handovers. The BSC is the connection between the Mobile Station and Mobile Switching Center.

- Better voice quality

- Low-cost alternatives to making calls, such as the Short message service

- $\quad$ Ease of deploying equipment from any vendors that implement the standard

- Offer roaming services so that subscribers can use their phones on GSM networks all over the world

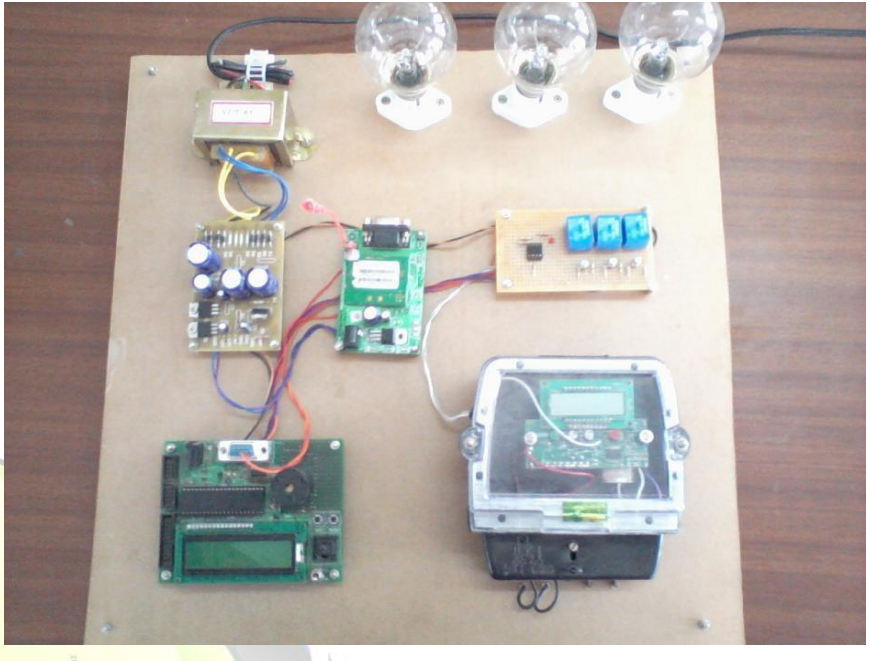

Fig.4. Layout of System Placed At Home

\section{IV.CONCLUSION}

In - this project an automatic meter reading system is designed using GSM Technology. The embedded micro controller is interfaced with the GSM Module. This setup is fitted in home. The energy meter is attached to the micro controller. This controller reads the data from the meter output and transfers that data to GSM Module through the serial port. The embedded micro controller has the knowledge of sending message to the system through the GSM module. Another system is placed in EB office, which is the authority office. When they send "unit request" to the microcontroller which is placed in home. Then the unit value is sent to the EB office PC through GSM module. According to the readings, the authority officer will send the information about the bill to the customer. If the customer doesn't pay bill on-time, the power supply to the corresponding home power unit is cut, by sending the command through to the microcontroller. Once the payment of bill is done the power supply is given to the customer. Power management concept is introduced, in which during the restriction mode only limited amount of power supply can be used by the customer. 


\section{REFERENCES}

[1]. Tariq Jamil, 2008 "Design and Implementation of a Wireless Automatic Meter Reading System" in Proceedings of the World Congress on Engineering.

[2]. Tarek Khalifa, Kshirasagar Naik and Amiya Nayak, "A Survey of Communication Protocols for Automatic Meter Reading Applications" in Communications Surveys \& Tutorials, IEEE.

[3]. Vinu V Das," Wireless Communication System for Energy Meter Reading" in 2009, International Conference on Advances in Recent Technologies in Communication and Computing.

[4]. Xiujie Dong, Yan Yang, You Zhou 2010 IEEE,“ The Design of Wireless Automatic Meter Reading System Based on SOPC" in WASE International conference on Information Engineering.

[5]. International Standards for all electrical, electronic and related technologies available at http://www.iec.ch.

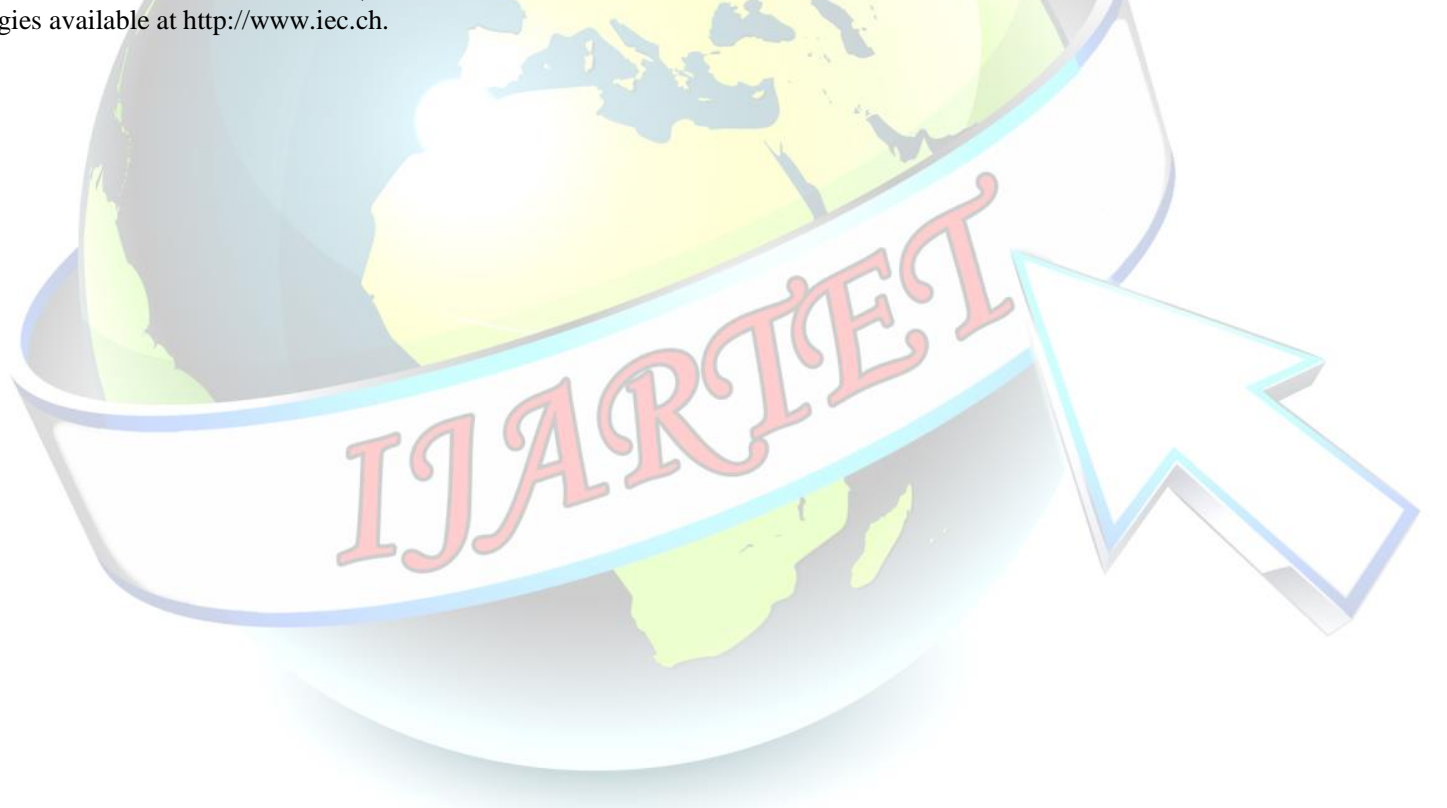

